\title{
ENGEVISTA
}

Página da revista: http://www.uff.br/engevista/seer/

\section{Análise de sensibilidade aplicada a um reator de redução catalítica seletiva Sensibility analysis applied for a reactor of selective catalytic reduction}

\author{
Marteson Cristiano dos Santos Camelo ${ }^{1}$ \\ Sergio Lucena ${ }^{2}$ \\ João Rui Barbosa de Alencar ${ }^{3}$ \\ Rony Glauco Melo ${ }^{4}$
}

Resumo: Neste trabalho modelou-se e simulou-se a dinâmica do leito de redução catalítica do $\mathrm{NO}_{\mathrm{X}}$, chamado leito RCS. Este leito constitui reatores de unidades de abatimento de emissões atmosféricas, como o do processo SNOX da Haldor Topsoe ${ }^{\circledR}$, que é constituído por dois leitos catalíticos, o primeiro providencia a redução de $\mathrm{NO}_{x}$, e o segundo a oxidação do $\mathrm{SO}_{2}$. Como parte do estudo de modelagem e simulação do leito RCS técnicas de análise de sensibilidade global foram aplicadas determinando-se a influência de alguns parâmetros sobre a concentração de $\mathrm{NO}, \mathrm{NH}_{3}$ e $\mathrm{SO}_{3}$, na saída do reator. Nesta análise verificou-se que os parâmetros de maior influência sobre as concentrações de $\mathrm{NO}$ e $\mathrm{NH}_{3}$ são o teor de $\mathrm{NO}$ na corrente de alimentação, o comprimento do canal monolítico e a razão molar $\mathrm{NH}_{3} / \mathrm{NO}$. Entretanto, a concentração de $\mathrm{SO}_{3}$ é fortemente influenciada pela temperatura de alimentação do reator.

Palavras-chave: Reator SCR, análise de sensibilidade, modelagem matemática, redução do $\mathrm{NO}_{\mathrm{X}}$.

\footnotetext{
${ }^{1}$ UFERSA - Universidade Federal Rural do Semi-Árido

2 UFPE - Universidade Federal de Pernambuco

3 Petrobras

${ }^{4}$ IFPE - Instituto Federal de Educação, Ciência e Tecnologia de Pernambuco
}

ENGEVISTA, V. 19, n.1 , p. 166-184, Janeiro 2017. 


\begin{abstract}
In this paper is modeled and simulated the dynamics of a bed catalytic $\mathrm{NO}_{\mathrm{X}}$ reduction, called SCR bed. This bed has on units of atmospheric emissions abatement, such as the Haldor Topsoe ${ }^{\circledR}$ SNOX process , which consists of two catalyst beds, the first provides the $\mathrm{NO}_{\mathrm{X}}$ reduction and the second one provides the oxidation of $\mathrm{SO}_{2}$. As part of the study, global sensitivity analysis techniques were applied to RCS bed. It was determined the influence of some parameters on the concentration of $\mathrm{NO}, \mathrm{NH}_{3}$ and $\mathrm{SO}_{3}$ in the reactor outlet. On this analysis it was found that the most influential parameters on the concentrations of $\mathrm{NO}$ and $\mathrm{NH}_{3}$ are the NO content in the feed stream, the length of the monolithic channel and the molar ratio $\mathrm{NH}_{3} / \mathrm{NO}$. However, the concentration of $\mathrm{SO}_{3}$ is strongly influenced by the reactor feed temperature.
\end{abstract}

Keywords: SCR reactor, sensitivity analysis, mathematical modeling, $\mathrm{NO}_{\mathrm{X}}$ reduction. 


\section{Introdução}

De acordo com o balanço energético nacional de 2014, aproximadamente $60 \%$ de toda energia produzida no Brasil em 2013 teve como fonte combustíveis fósseis, acarretando num grande passivo ambiental já que a queima desses combustíveis produz diversos tipos de poluentes como $\mathrm{SO}_{\mathrm{X}}, \mathrm{NO}_{\mathrm{X}}$ e material particulado. Estes poluentes têm seus limites de emissão definidos pela resolução CONAMA 382, para o $\mathrm{NO}_{x}$ essa resolução impõe como limite uma concentração de $200 \mathrm{mg} / \mathrm{Nm}^{3}$, em caldeiras ou fornos queimando gás de refinaria que possuam potência maior que $70 \mathrm{MW}$.

Essa restrição motivou a Petrobras ${ }^{\circledR}$ a investir em uma unidade de abatimento de emissões atmosféricas, como por exemplo, o processo SNOX da Haldor Topsoe ${ }^{\circledR}$, que terá sua primeira unidade instalada no Brasil na Refinaria Abreu e Lima (Ipojuca-PE, Brasil) (RNEST). Devido a sua especificidade para processar petróleos pesados, e em razão dos componentes presentes, esta refinaria irá gerar um alto grau de contaminantes incluindo principalmente $\mathrm{NO}_{\mathrm{X}}$ e $\mathrm{SO}_{2}$.

A unidade SNOX possui um reator constituído por dois leitos catalíticos. O primeiro é responsável pela redução catalítica do $\mathrm{NO}_{\mathrm{X}}$, e no segundo ocorrem reações de oxidação do $\mathrm{SO}_{2}$.

Segundo Forzatti et al. (2001) a redução catalítica seletiva (RCS) é um dos processos mais desenvolvidos e empregados mundialmente no tratamento de gases efluentes contendo $\mathrm{NO}_{\mathrm{X}}$, devido a sua eficiência, seletividade e economia. A aplicação do processo RCS se aplica desde o tratamento de gases advindos da combustão de plantas industriais como pode ser visto em Dhanushkodi et al. (2008), Nguyen et al. (2010), Tronconni et al. 1996, Chen et al. 2012. Até mesmo no controle das emissões de $\mathrm{NO}_{\mathrm{X}}$ em motores a Diesel Faghihi (2010), Depcik et al. (2005), Ruggeri et al. (2012).

A reação global para o processo RCS é representada pela equação 1, a qual ocorre em temperaturas entre $250^{\circ} \mathrm{C}$ e $450^{\circ} \mathrm{C}$, com oxigênio em excesso. $\mathrm{O}$ termo seletivo é aplicado, pois a amônia reage diretamente com o $\mathrm{NO}_{\mathrm{x}}$ ao invés de ser oxidada pelo oxigênio, de acordo com a equação 2.

$$
\begin{gathered}
4 \mathrm{NO}+4 \mathrm{NH}_{3}+\mathrm{O}_{2} \rightarrow 4 \mathrm{~N}_{2}+6 \mathrm{H}_{2} \mathrm{O} \\
4 \mathrm{NH}_{3}+3 \mathrm{O}_{2} \rightarrow 2 \mathrm{~N}_{2}+6 \mathrm{H}_{2} \mathrm{O}
\end{gathered}
$$

Lietti et al (2000) verificou que o $\mathrm{SO}_{2}$ presente nos gases efluentes pode ser oxidado a $\mathrm{SO}_{3}$, e este, por sua vez, reage com amônia e água produzindo sulfato de amônio, o qual reduz a eficiência do reator RCS, provocando problemas de corrosão.

Através da modelagem matemática do processo é possível analisar a planta em vários cenários, como por exemplo, nas paradas e retomadas da operação, assim como variações na carga 
de alimentação. Cada um desses cenários gera uma entrada característica para o modelo e consequentemente uma saída diferente para o modelo. Um estudo mais detalhado sobre a importância que as entradas do processo têm sobre a saída pode ser realizado através da análise de sensibilidade das variáveis que compõem o modelo matemático.

Um dos métodos utilizados para análise de sensibilidade é a partir da derivada $\frac{\partial Y_{i}}{\partial X_{i}}$, em que $\mathrm{Y}$ representa a saída de um determinado processo e $\mathrm{X}$ representa o fator de entrada. Este tipo de abordagem é classificada como análise de sensibilidade local e tem como grande vantagem a sua eficiência computacional, por necessitar que o modelo matemático seja resolvido poucas vezes para se obter a sensibilidade do modelo. Porém segundo Saltelli et al. (2012) o método falha quando o modelo em estudo não é linear e quando são realizadas grandes desvios na entrada de referência do modelo. Uma alternativa para lidar com tais limitações é a análise de sensibilidade global, que permite o estudo de modelos não lineares, assim como analisar a resposta do sistema em todo o intervalo de variação dos parâmetros de entrada do processo.

Este trabalho tem como objetivo modelar e simular a dinâmica do leito RCS. E aplicar técnicas de análise de sensibilidade global visando determinar a influência que alguns parâmetros possuem sobre a concentração de $\mathrm{NO}, \mathrm{NH}_{3}$ e $\mathrm{SO}_{3}$, na saída do reator.

\section{Metodologia}

\subsection{Descritivo do processo}

Uma unidade de abatimento de emissões atmosféricas do tipo SNOX consiste no tratamento dos gases de combustão com o objetivo de remover material particulado, óxidos de nitrogênio e dióxido de enxofre. O reator da unidade de abatimento de emissões atmosféricas, a ser instalado na RNEST, é dividido em dois leitos catalíticos, nos quais ocorrem as reações de remoção de $\mathrm{NO}_{\mathrm{X}}$ e $\mathrm{SO}_{2}$. A carga que alimenta o reator passa pelo leito RCS, onde ocorre o qual é a redução catalítica do $\mathrm{NO}_{\mathrm{x}}$ a $\mathrm{N}_{2}$, além desta reação também pode ocorrer nesse leito a oxidação do $\mathrm{SO}_{2}$ a $\mathrm{SO}_{3}$. O leito RCS é constituído por blocos catalíticos de estrutura monolítica, que pode apresentar diferentes compostos ativos como, por exemplo, $\mathrm{V}_{2} \mathrm{O}_{5} / \mathrm{TiO}_{2}, \mathrm{~V}_{2} \mathrm{O}_{5} \mathrm{Ti}, \mathrm{V}_{2} \mathrm{O}_{5} / \mathrm{WO}_{3} / \mathrm{TiO}_{2}$.

A corrente de saída do primeiro leito segue para o segundo onde ocorre a oxidação do $\mathrm{SO}_{2} \mathrm{a} \mathrm{SO}_{3}$. Por fim, o efluente gasoso tratado, rico em $\mathrm{SO}_{3}$, troca calor com a corrente de entrada do reator, para melhorar a eficiência energética do processo, e segue para o condensador onde é resfriado até uma temperatura inferior ao ponto de orvalho do ácido sulfúrico, através de uma corrente de ar. Neste equipamento o $\mathrm{SO}_{3}$ reage com a água contida na carga de alimentação produzindo ácido sulfúrico. Mais detalhes sobre o processo SNOX podem ser vistos em Alencar e Boas (2012). 


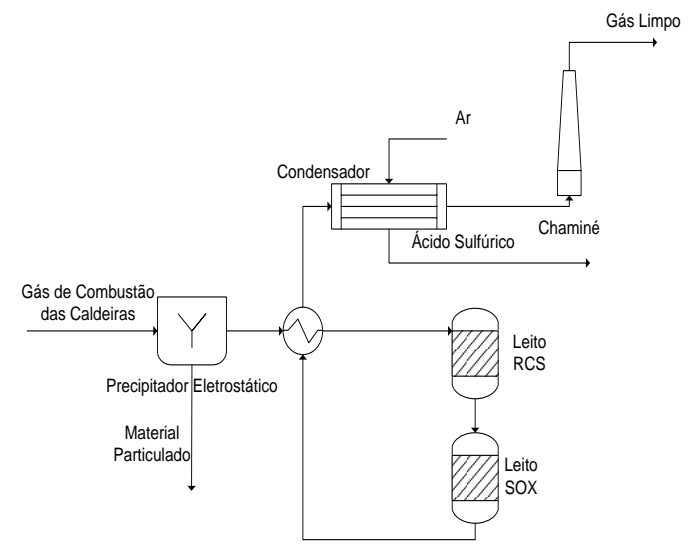

Figura 1. Fluxograma simplificado na seção de reação SNOX.

\subsection{Modelagem matemática}

O leito RCS é composto por vários blocos de catalisadores monolíticos, estes possuem canais geralmente com geometria retangular. Portanto, a seção transversal do reator RCS vai ser formada por diversos canais, cada um desses canais têm suas paredes revestidas pelo material catalítico, no qual o $\mathrm{NO}$ e o $\mathrm{NH}_{3}$ irão ser adsorvidos e reagir. Uma grande vantagem desse tipo de leito catalítico é a pequena perda de carga principalmente quando comparado ao leito fixo. As considerações feitas para modelagem do leito RCS são mostradas a seguir:

- Um único canal pode representar todo comportamento do leito

- Escoamento laminar

- Não há acúmulo de massa na fase sólida

- Escoamento unidirecional, ou seja, apenas na direção axial.

- Queda de pressão desprezível ao longo do leito

- Condução de calor axial na fase sólida desprezível

- Troca de calor entre o reator e o ambiente desprezível.

Os balanços de massa e energia na fase gás são mostrados respectivamente pelas equações 3 e 4, na equação 4 o índice " $i$ " representa os compostos $\mathrm{NH}_{3}$ ou $\mathrm{NO}$.

$$
\begin{gathered}
\frac{\partial T_{g}}{\partial t}=-u \frac{\partial T_{g}}{\partial z}-\frac{h S_{v}}{\varepsilon \rho_{g} c_{p g}}\left(T_{g}-T_{s}\right) \\
\frac{\partial C_{g, i}}{\partial t}=-u \frac{\partial C_{g, i}}{\partial z}-k_{m, i} S_{v}\left(C_{g, i}-C^{*}{ }_{i}\right)
\end{gathered}
$$

O balanço de massa e energia na fase sólida são respectivamente as equações 5, 6 e 7. O balanço para a superfície coberta por amônia é dado pela equação 8 , na qual $\theta_{N H 3}$ é o valor médio 
de amônia adsorvida na superfície catalítica, a qual depende das taxas de: reação do NO, adsorção e dessorção de amônia. $\mathrm{O}$ balanço de energia na fase sólida é dado pela equação 8.

$$
\begin{gathered}
\frac{k_{m, N H_{3}}}{a}\left(C_{g, N H_{3}}-C_{s, N H_{3}}\right)-r_{a d s}+r_{d e s} \\
\frac{k_{m, N O}}{a}\left(C_{g, N O}-C_{s, N O}\right)-r_{N O} \\
\frac{\partial T_{s}}{\partial t}=\frac{S_{v} h}{\rho_{s} c_{p s}}\left(T_{g}-T_{s}\right)+\frac{\sum_{j} \Delta H_{j} R_{j}}{\rho_{s} c_{p s}} \\
\Omega_{N H 3} \frac{\partial \boldsymbol{\theta}_{N H 3}}{\partial \boldsymbol{t}}=\boldsymbol{r}_{\boldsymbol{a d s}}-\boldsymbol{r}_{\text {des }}-\boldsymbol{r}_{N \boldsymbol{O}}
\end{gathered}
$$

Também foi considerado na modelagem a reação de oxidação do $\mathrm{SO}_{2}$ a $\mathrm{SO}_{3}$ no leito catalítico. Segundo Forzatti et al. (1999) a conversão do $\mathrm{SO}_{2}$ é limitada a aproximadamente $1 \%$, portanto a concentração deste pode ser considerada constante ao longo do reator. E apenas os balanços para $\mathrm{o} \mathrm{SO}_{3}$ nas fases gás, equação 09, e sólida, equação 10, são necessários.

$$
\begin{gathered}
\frac{\partial C_{\mathrm{SO}_{3}, i}}{\partial t}=-u \frac{\partial C_{\mathrm{SO}_{3}, i}}{\partial z}-\frac{1-\varepsilon}{\varepsilon}\left(r_{a d s, \mathrm{SO}_{3}}-r_{d e s, \mathrm{SO}_{3}}\right) \\
\frac{\partial \theta_{\mathrm{SO}_{3}}}{\partial t}=r_{a d s, \mathrm{SO}_{3}}-r_{d e s, \mathrm{SO}_{3}}+r_{\mathrm{SO}_{2}}
\end{gathered}
$$

O coeficiente de transferência de massa foi calculado através da equação 11 , pois conforme Santos et al. (1998) dentre várias correlações para cálculo do coeficiente de transferência de massa em leitos monolíticos testadas, esta é a que melhor se adequou aos dados experimentais. Já o coeficiente de transferência de calor foi calculado pela equação 12, a qual foi obtida em Votruba et al. (1975).

$$
\begin{gathered}
k_{m, i}=\frac{D_{i}}{d_{h}} 0,705 S c_{i}^{0.56}\left(\operatorname{Re} \frac{d_{h}}{L}\right)^{0.43} \\
h=\frac{k}{d_{h}} 0.5071\left(\operatorname{Re} \frac{d_{h}}{L}\right)^{\frac{2}{3}}
\end{gathered}
$$

A cinética utilizada no modelo foi a proposta por Lietti et al (2000).

As equações diferenciais parciais que compõem o modelo matemático foram resolvidas através do método das linhas e a variável espacial, z, foi discretizada. O conjunto de equações resultantes gerou um sistema de equações diferenciais algébricas, este foi resolvido através do 
solver ODE15s do Matlab ${ }^{\circledR}$, que além de resolver sistemas de equações diferenciais algébricas possui passo de integração variável.

As condições iniciais e de contorno são mostradas abaixo.

$$
\begin{aligned}
& z=0 \\
& C_{g, i}=C_{s, i}=C_{i}^{0} \\
& i=\mathrm{NO}, \mathrm{NH}_{3}, \mathrm{SO}_{3} \\
& T_{s}=T_{g}=T_{0} \\
& \theta_{i}=0 \quad i=\mathrm{NH}_{3}, \mathrm{SO}_{3} \\
& C_{g, i}=C_{s, i}=C_{i}^{0} \\
& i=\mathrm{NO}, \mathrm{NH}_{3}, \mathrm{SO}_{3} \\
& t=0 \\
& T_{s}=T_{g}=T_{0} \\
& \theta_{i}=0 \quad i=\mathrm{NH}_{3}, \mathrm{SO}_{3}
\end{aligned}
$$

\subsection{Análise de sensibilidade}

Os métodos de análise de sensibilidade investigam a saída dos modelos como uma função dos parâmetros. Nestes métodos a dificuldade fundamental é a garantia de que o intervalo de variação dos parâmetros ou variáveis de entrada será investigado, e como eles devem funcionar de forma combinada. Assim, uma amostragem baseada na análise de sensibilidade é uma amostragem em que o modelo é executado repetitivamente testando-se várias combinações dos valores amostrados a partir da distribuição dos fatores de entrada.

Saltelli et al. (2004) divide os métodos de análise de sensibilidade em Screening, Local e Global. Dentre esses, destaca-se a análise de sensibilidade global por possibilitar que os efeitos da variação de todos os parâmetros na entrada sejam verificados, sendo permitido que estes parâmetros variem sob seus intervalos, levando em consideração a forma de suas funções de densidade de probabilidade.

Uma classe de métodos globais de interesse são os métodos baseados em variância, que começaram a ser desenvolvidos em 1970 com o objetivo de lidar com modelos não lineares, como por exemplo, processos químicos. Saltelli et.al (2004), desenvolveu uma metodologia para calcular a sensibilidade global a partir da decomposição de variância. Enquanto, Saltelli et.al (2012) faz uma revisão sobre a análise de sensibilidade em modelos químicos e também mostra a aplicação de diferentes métodos globais a um reator químico. 
A técnica de decomposição de variância divide a variância de saída em partes, devido aos fatores, ou variáveis, de entrada e suas combinações. Seja uma função integrável definida de $(0,1)$.

$$
\begin{gathered}
f(X)=f_{0}+\sum_{i=1}^{n} f_{i}\left(X_{i}\right)+\sum_{i<j}^{n} f_{i}\left(X_{i}, X_{j}\right) \\
+\cdots+f_{12 . . n}(X)
\end{gathered}
$$

De acordo com Sobol (2001) os membros da equação 13 são ortogonais, assim a decomposição de variância é dada pela equação 14. Em que, $Y$ representa a saída e $n$ o número de parâmetros de entrada, do modelo.

$$
\begin{array}{r}
\operatorname{Var}(Y)=\sum_{i=1}^{n} D_{i}(Y)+\sum_{i<j}^{n} D_{i j}(Y) \\
+\cdots+D_{12 \ldots n}(Y)
\end{array}
$$

Ao dividir-se a equação 14, pela variância de $Y$ são obtidos os índices de sensibilidade. Portanto, o primeiro termo do lado direito é o índice de primeira ordem e representa o efeito principal que cada parâmetro de entrada tem sobre a variância de saída. Além do índice de primeira ordem, a equação 14 mostra índices de ordem superior, $D_{i j}$, estes quantificam os efeitos que iterações nos parâmetros de entrada têm sobre a saída do modelo. Porém, o cálculo dos índices de ordem superior demandam um esforço computacional elevado, já que para o cálculo desses índices o número de simulações do modelo se torna elevado. Uma alternativa para quantificar o efeito que a iteração dos parâmetros de entrada possui sobre a variância de saída é o índice de sensibilidade total, que foi definido em Saltelli (2002), e é mostrado na equação 15.

$$
S_{i}^{T}=S_{i}+S_{i j}+\cdots+S_{12 d}
$$

Saltelli et al. (2012) mostra em seu trabalho que o número de simulações do modelo para cálculo do índice total e de primeira ordem é $(n+2) N$, em que $N$ é o número de amostras e $n$ o número de parâmetros de entrada, já para o cálculo do índice de segunda ordem o número de simulações necessárias seria $(2 n+2) N$. Com o propósito de calcular a variância de saída do modelo são definidas 3 matrizes, $A, B$ e $C^{i}$, em que cada linha representa uma amostra com diferentes valores para os parâmetros de entrada e cada coluna é um parâmetro diferente que está sendo avaliado no estudo. As matrizes de entrada devem ser construídas de modo a garantir que os pontos de amostragem sejam distribuídos uniformemente no espaço de entrada do modelo, as matrizes $A$ e $B$ são geradas a partir de métodos de Monte Carlo, neste trabalho foi utilizado o Latin Hypercube. Já a matriz $C^{i}$ contém todas as colunas da matriz B exceto a coluna $i$, a qual é modificada pela coluna correspondente da matriz $A$. 
A partir dos dados gerados pelas matrizes de entrada a sensibilidade total e de primeira ordem pode ser calculada respectivamente pelas equações, 16 e 17, as quais segundo Sobol et al. (2007) permitem o cálculo da sensibilidade do modelo com maior exatidão e também reduzem o número de simulações necessárias para obter um valor preciso.

$$
\begin{gathered}
S_{i}=\frac{\frac{1}{N} \sum_{j=1}^{N} y_{A_{j}} y_{C_{j}}^{i}-\frac{1}{N} \sum_{j=1}^{N} y_{A_{j}} y_{B_{j}}}{\frac{1}{N} \sum_{j=1}^{N} y_{A_{j}}^{2}-\frac{1}{N} \sum_{j=1}^{N} y_{A_{j}} y_{B_{j}}} \\
S_{i}^{T}=1-\frac{\frac{1}{N} \sum_{j=1}^{N} y_{B_{j}} y_{C}^{i}-\frac{1}{N} \sum_{j=1}^{N} y_{A_{j}} y_{B_{j}}}{\frac{1}{N} \sum_{j=1}^{N} y_{A_{j}}^{2}-\frac{1}{N} \sum_{j=1}^{N} y_{A_{j}} y_{B_{j}}}
\end{gathered}
$$

Os parâmetros analisados foram: o comprimento do elemento catalítico (L), a pressão no reator $(\mathrm{P})$, a razão amônia óxido de nitrogênio $(\alpha)$, a temperatura de alimentação $\left(T_{0}\right)$, vazão de entrada, teor de $\mathrm{NO}$ e teor de $\mathrm{SO}_{2}$ na entrada. Dentre esses apenas L se classifica como parâmetro de projeto, todos os demais são parâmetros operacionais do reator.

Admitiu-se uma função de probabilidade uniforme para representar L, sendo utilizado o intervalo $0,15 \leq L \leq 1,5$, enquanto para os parâmetros operacionais admitiu-se uma distribuição normal com média e desvio padrão dados na Tabela 1, cujos dados foram inseridos num código computacional em ambiente Matlab $^{\circledR}$ e através das funções lhsdesign e lhsnorm, pertencentes ao Matlab $^{\circledR}$, foram gerados conjuntos de dados de 1000 pontos para compor as matrizes $A, B$ e $C^{i}$. Cada uma delas tem 1000 linhas e 7 colunas.

Tabela 1. Intervalo de variação dos parâmetros de entrada.

\begin{tabular}{ccc}
\hline Parâmetro & Média & Desvio Padrão \\
Vazão $\left(\mathrm{Nm}^{3} / \mathrm{h}\right)$ & $5 \times 10^{5}$ & $5 \times 10^{5}$ \\
$T_{0}\left({ }^{\circ} \mathrm{C}\right)$ & 350 & 27 \\
$P(\mathrm{~atm})$ & 1,5 & 0,14 \\
Teor $\mathrm{NO}(\mathrm{ppm})$ & 300 & 60 \\
Teor $\mathrm{SO}_{2}(\mathrm{ppm})$ & 1500 & 400 \\
$\alpha$ & 1 & 0,17 \\
\hline
\end{tabular}




\section{Resultados}

A simulação do reator levou em consideração as condições operacionais mostradas na Tabela 2, o resultado foi comparado com os resultados experimentais de Tronconi et al. (1998).

Tabela 2. Parâmetros de entrada do reator RCS.

\begin{tabular}{lc}
\hline Condição operacional & Valor \\
\hline Temperatura de & $360{ }^{\circ} \mathrm{C}$ \\
alimentação & \\
Teor NO na entrada & $560 \mathrm{ppm}$ \\
Teor $\mathrm{SO}_{2}$ na entrada & $1000 \mathrm{ppm}$ \\
$\mathrm{AV}$ & $33 \mathrm{~m} / \mathrm{h}$ \\
Pressão & $1,5 \mathrm{~atm}$ \\
\hline \multicolumn{2}{c}{ Fonte: Tronconi et al. $(1998)$}
\end{tabular}

Fonte: Tronconi et al. (1998)

A Figura 2 mostra a variação na concentração de saída de $N O$ ao longo do tempo, esta figura representa um experimento em que a alimentação de $\mathrm{NH}_{3}$ é interrompida repentinamente. O reator foi alimentado inicialmente com uma concentração inicial de amônia correspondente ao valor de $\alpha$, e em $t=500 \mathrm{~s}$ a concentração de $\mathrm{NH}_{3}$ na alimentação foi igualada a zero, o que resultou no aumento da concentração de saída do NO para o mesmo valor da sua concentração de entrada. Observou-se que a concentração de NO obtida como saída do modelo se ajustou bem aos dados experimentais, para diferentes valores de $\alpha$.

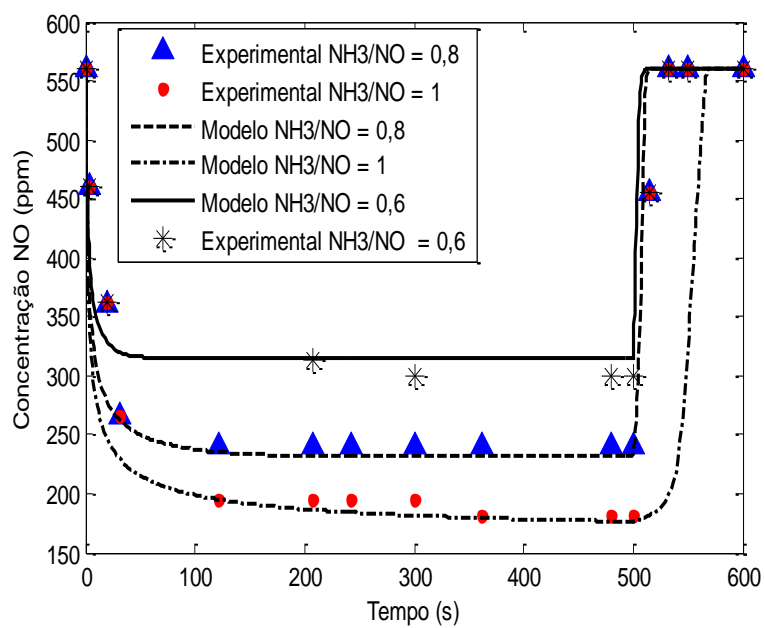

Figura 2. Perfis de concentração de NO ao longo do tempo na saída do reator.

Após simular o modelo com os parâmetros de entrada, contidos nas matrizes $A, B$ e $C^{i}$, foi calculado os índices de sensibilidade total e de primeira ordem referentes as seguintes saídas do modelo: concentração de $\mathrm{NO}$, concentração de $\mathrm{NH}_{3}$ e concentração de $\mathrm{SO}_{3}$.

Dentre os parâmetros avaliados o que teve maior influência na concentração de NO na saída do reator foi o $\alpha$, seguida por L e o teor de NO, Tabela 03. Isto mostra que a concentração de NO na saída do reator está relacionada a concentração de alimentação de $\mathrm{NH}_{3}$, já que esta 
variável está vinculada a $\alpha$. A pressão e o teor de $\mathrm{SO}_{3}$ na alimentação tiveram seus índices de primeira ordem iguais a zero, apesar disso para concluir que esses parâmetros são não influentes é necessário também que o índice de sensibilidade total seja igual a zero, o que não acorre neste caso. Como o valor de $S_{i}^{T}$ é maior que zero significa que a influência que esses parâmetros tem na concentração de saída de NO está relacionada a interação desses com os demais parâmetros de entrada do modelo.

Tabela 3. Índices de sensibilidade total e de primeira ordem para Concentração de NO.

\begin{tabular}{cll}
\hline Parâmetro & $\boldsymbol{S}_{\boldsymbol{i}}(\mathbf{s})$ & $\boldsymbol{S}_{\boldsymbol{i}}^{\boldsymbol{T}} \mathbf{( \mathbf { s } )}$ \\
$L$ & 0,27 & 0,28 \\
Vazão & 0,02 & 0,04 \\
$T_{0}$ & 0,01 & 0,03 \\
$P$ & 0 & 0,02 \\
Teor $\mathrm{NO}$ & 0,06 & 0,12 \\
Teor $\mathrm{SO}_{2}$ & 0 & 0,02 \\
$\alpha$ & 0,58 & 0,64 \\
\hline
\end{tabular}

A Figura 3 mostra que os índices de sensibilidade de primeira ordem relacionados a concentração de $N O$ variam ao longo do reator. Nesta figura é possível avaliar que na entrada do reator o teor de $N O$ tem maior influência na concentração de $N O$ do que os demais parâmetros, porém esta influência diminui ao longo do reator devido a reação de consumo do $N O$. Este efeito causado pela reação é justificado pelo fato de que ambos $\alpha$ e L passarem a ter maior importância à medida que se está mais próximo a saída do leito. Já que o comprimento do reator afeta na área disponível para transferência de massa, e a concentração de amônia está relacionada a estequiometria da reação de redução do $N O$. 


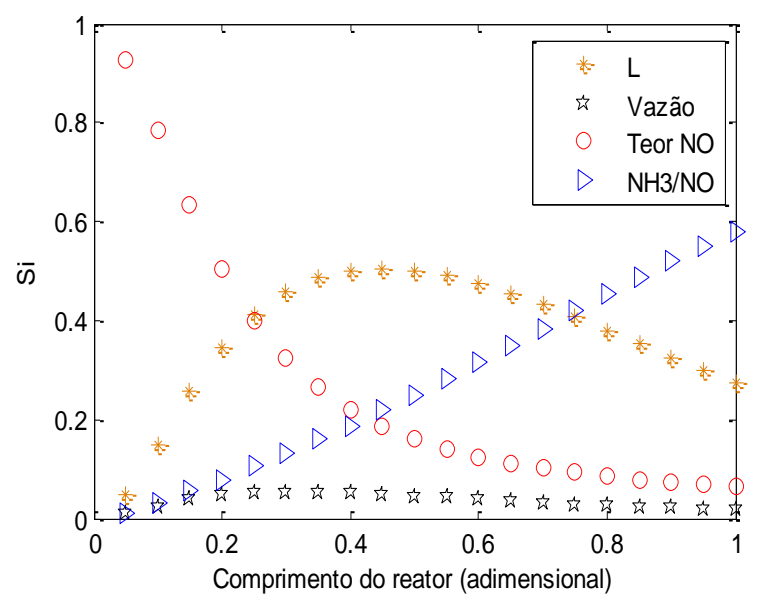

Figura 3. Índice de sensibilidade de primeira ordem relacionado a concentração de NO ao longo do reator.

Como pode ser visto na Tabela 4, o parâmetro mais influente na concentração de saída do $\mathrm{NH}_{3}$ é o L, como discutido anteriormente esse comportamento era esperado já que $\mathrm{L}$ tem interferência direta na área disponível para transferência de massa. Além desse parâmetro apenas o teor de $\mathrm{NO}$ e $\alpha$ tiveram influência na concentração de saída de $\mathrm{NH}_{3}$, pois os demais parâmetros tiveram $S_{i}$ e $S_{i}^{T}$ iguais a zero.

Tabela 4. Índices de sensibilidade total e de primeira ordem para Concentração de $\mathrm{NH}_{3}$.

\begin{tabular}{ccc}
\hline Parâmetro & $\boldsymbol{S}_{\boldsymbol{i}}$ & $\boldsymbol{S}_{\boldsymbol{i}}^{\boldsymbol{T}}$ \\
$L$ & 0,85 & 0,81 \\
Vazão & 0 & 0 \\
$T_{0}$ & 0 & 0 \\
$P$ & 0 & 0 \\
Teor $\mathrm{NO}$ & 0,02 & 0,02 \\
Teor $\mathrm{SO}_{2}$ & 0 & 0 \\
$\alpha$ & 0,02 & 0,02
\end{tabular}

Diferente do caso discutido anteriormente, que a influência de $\alpha$ aumentou ao longo do leito, houve uma diminuição na importância desse parâmetro, Figura 4. Pois as taxas de adsorção e dessorção, que são os termos do modelo matemático que mais afetam a concentração de $\mathrm{NH}_{3}$ ao longo do reator, independem da concentração inicial de amônia, a qual tem relação direta com $\alpha$. 


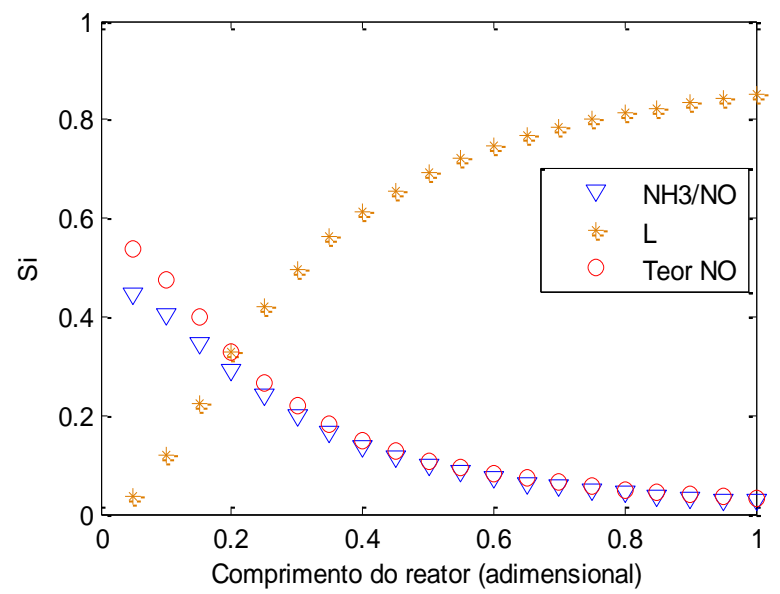

Figura 4. Índice de sensibilidade de primeira ordem relacionado a concentração de $\mathrm{NH}_{3}$ ao longo do reator.

A Tabela 5 mostra que $S_{T_{0}}$ foi maior que os demais valores de $S_{i}$, logo, a temperatura de alimentação do reator é a variável que tem maior influência sobre a concentração de saída referente ao $\mathrm{SO}_{3}$. Comparado as demais concentrações analisadas essa foi a que apresentou maior dependência com $T_{0}$, o que pode ser explicado pela característica da cinética dessa reação. Já a vazão, o teor de NO e $\alpha$ tiveram valores nulos para o $S_{i}$. Entretanto, segundo Saltelli et al. (2008) esses parâmetros irão interferir na concentração de saída do $\mathrm{SO}_{3}$ por apresentarem $S_{i}^{T}$ não nulo, como pode ser visto na Figura 8, onde é mostrado o perfil de concentração de $\mathrm{SO}_{3}$ na saída do reator para diferentes valores de $\alpha$.

Tabela 5. Índices de sensibilidade total e de primeira ordem para Concentração de $\mathrm{SO}_{3}$.

$\begin{array}{ccc}\text { Parâmetro } & \boldsymbol{S}_{\boldsymbol{i}} & \boldsymbol{S}_{\boldsymbol{i}}^{\boldsymbol{T}} \\ \mathrm{L} & 0,08 & 0,21 \\ \text { Vazão } & 0 & 0,12 \\ T_{0} & 0,71 & 0,88 \\ P & 0,01 & 0,11 \\ \text { Teor } \mathrm{NO} & 0 & 0,11 \\ \text { Teor } \mathrm{SO}_{2} & 0,04 & 0,18 \\ \alpha & 0 & 0,15\end{array}$

Embora o valor de $S_{i}$ na saída do reator para a vazão e $\alpha$ tenha sido nulos observa-se na Figura 5, que próximo a região de entrada do reator esses índices não são nulos. No caso do $S_{\alpha} \mathrm{o}$ maior valor inicial está relacionado a cinética da reação de oxidação do $\mathrm{SO}_{2}$, uma vez que esta reação ocorre em paralelo a reação de redução do $N O$ nos sítios ativos do catalisador. Já o valor de $S_{L}$ permanece praticamente constante ao longo do reator. 


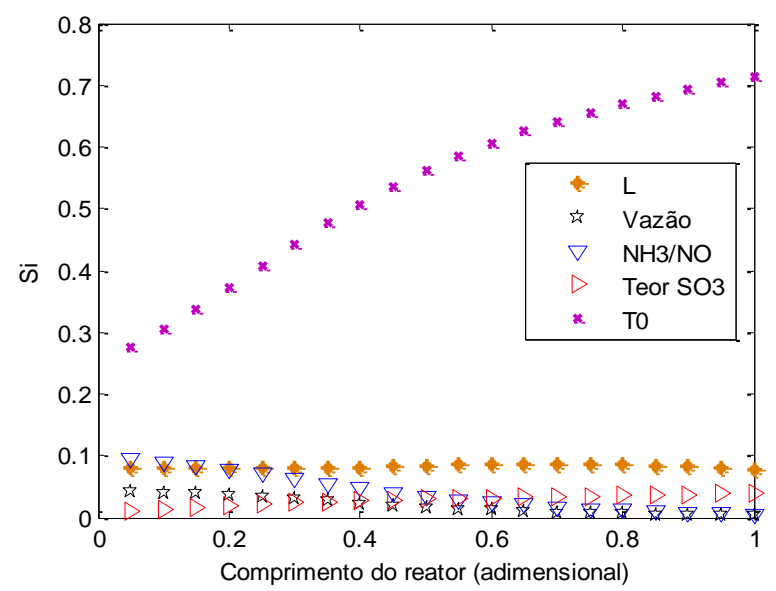

Figura 5. Índice de sensibilidade de primeira ordem relacionado a concentração de $\mathrm{SO}_{3}$ ao longo do reator.

Como o parâmetro operacional de maior influência nas concentrações de saída de $N O$ e $\mathrm{NH}_{3}$ foi $\alpha$. O comportamento dessas variáveis ao longo do tempo para diferentes valores de $\alpha$ foi simulado e é observado nas Figuras 6 e 7. Os demais parâmetros operacionais utilizados nessa simulação são dados na Tabela 6.

Tabela 6. Parâmetros operacionais utilizados na simulação.

\section{Parâmetro}

$$
\text { Vazão }\left(N m^{3} / h\right)
$$$$
T_{0}\left({ }^{\circ} \mathrm{C}\right)
$$$$
P(a t m)
$$

Teor NO (ppm)

Teor $\mathrm{SO}_{2}(\mathrm{ppm})$

\section{Valor}

$$
5 \times 10^{5}
$$

1500

A Figura 6 mostra o teor de $N O$ na saída do reator para diferentes valores de $\alpha$. Verificase que para $\alpha$ menor que um, há o aumento no teor de saída de $N O$ devido a menor quantidade de amônia disponível para reagir com o $N O$, presente na alimentação do reator, enquanto para $\alpha$ maior que um ocorre a diminuição no teor de $N O$ na saída. 


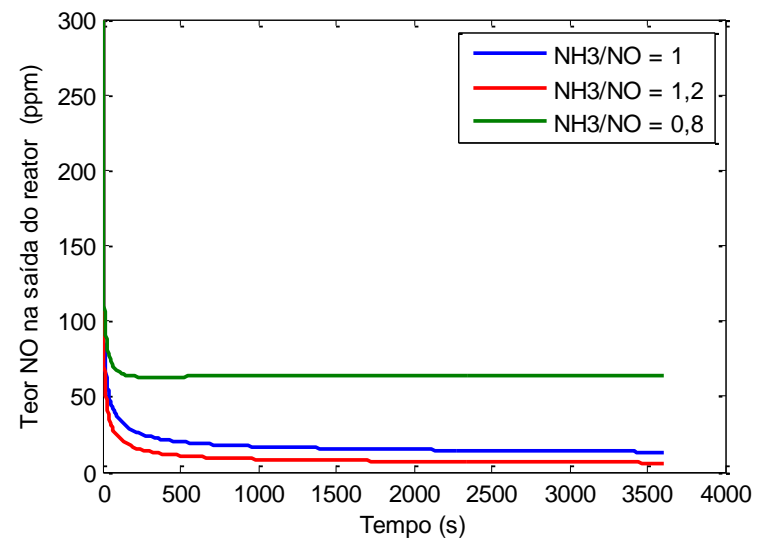

Figura 6. Teor de NO na saída do reator ao longo do tempo.

$\mathrm{O}$ teor de $\mathrm{NH}_{3}$ na saída do reator é apresentado nas Figuras 7 e 8 . O aumento em $\alpha$ ocasiona o aumento no teor de $\mathrm{NH}_{3}$ na saída, pois nessa condição há um pequeno excesso de amônia não reagida no reator. Porém, ao comparar-se a variação que ocorre no teor de $\mathrm{NH}_{3}$ e $\mathrm{NO}$ na saída do reator observa-se que a variação no teor de saída do $\mathrm{NH}_{3}$ é muito menor do que a que acontece no teor de $N O$, já que esse último sofre uma influência muito mais acentuada de $\alpha$, o que está de acordo com o que foi discutido anteriormente.

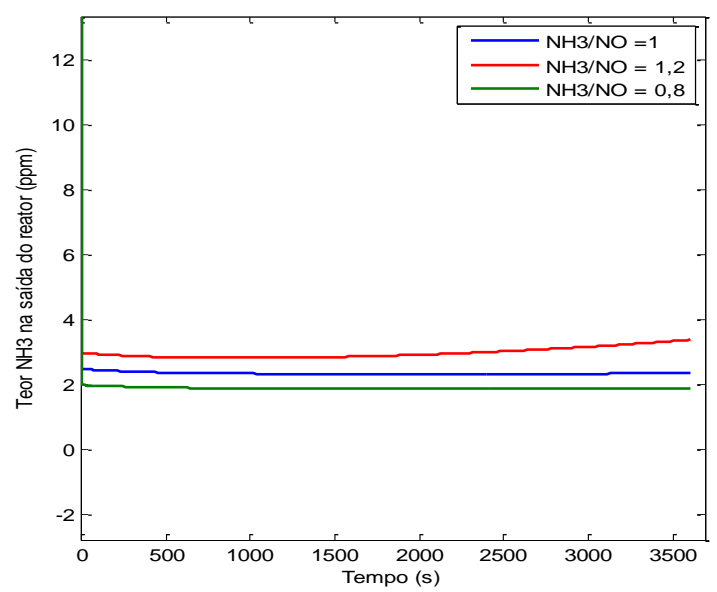

Figura 7. Teor de $\mathrm{NH}_{3}$ na saída do reator ao longo do tempo ampliado. 


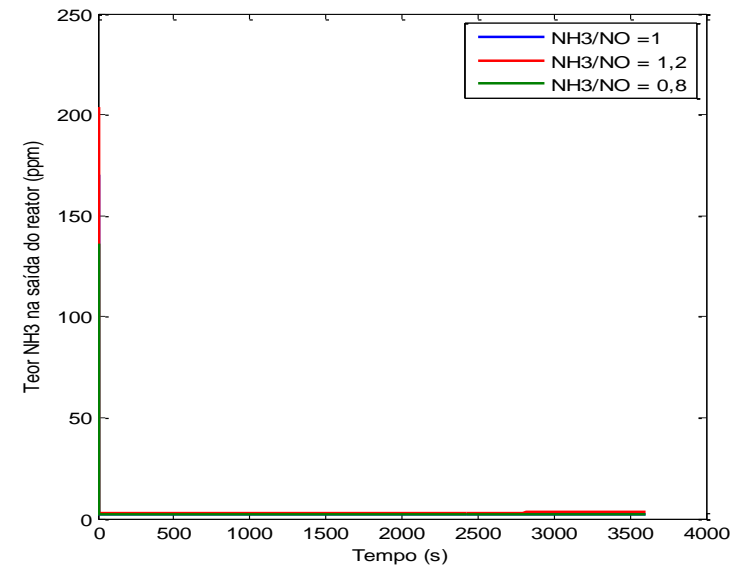

Figura 8. Teor de $\mathrm{NH}_{3}$ na saída do reator ao longo do tempo.

Na Figura 9 observa-se o comportamento do teor de saída do $\mathrm{SO}_{3}$ frente a variações no valor de $\alpha$, e como já era esperado mudanças no valor de $\alpha$ não acarretaram em alterações significativas no teor de saída do $\mathrm{SO}_{3}$, porém percebe-se que o aumento no valor de $\alpha$ conduz a uma pequena diminuição no valor dessa variável, devido ao efeito inibidor que a amônia tem nesta reação. De fato a variável mais influente no teor de saída do $\mathrm{SO}_{3}$ é $T_{0}$, como pode ser visto na Figura 10, onde $T_{0}$ conduziu a variações superiores no teor de saída do $\mathrm{SO}_{3}$, quando comparado a $\alpha$.

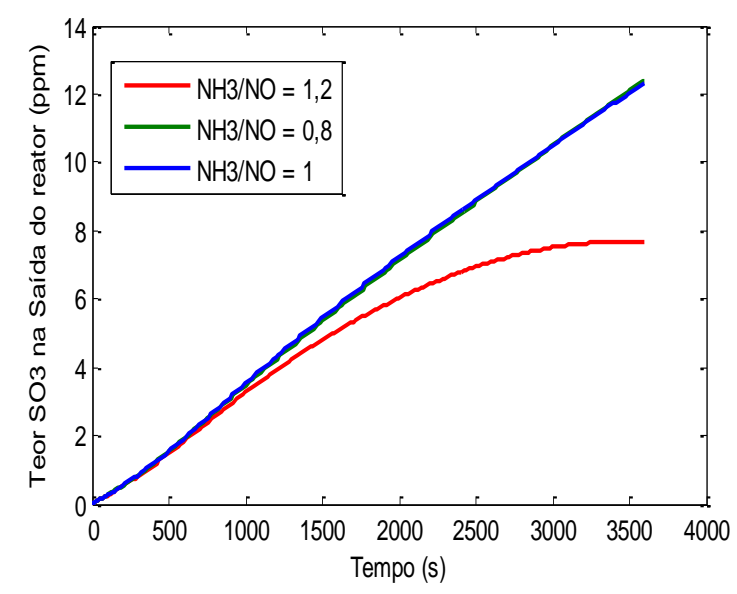

Figura 9. Teor de $\mathrm{SO}_{3}$ na saída do reator ao longo do tempo. 


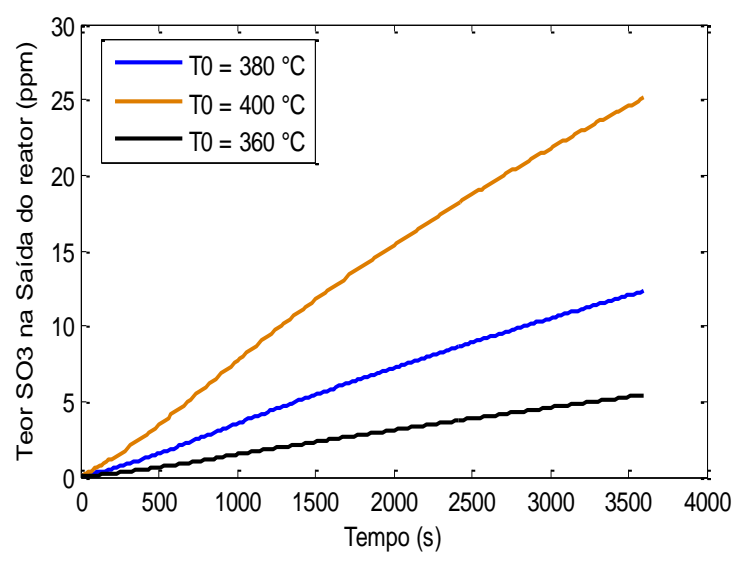

Figura 10. Teor de $\mathrm{SO}_{3}$ na saída do reator ao longo do tempo para diferentes valore de $\mathrm{T} 0$.

\section{Conclusões}

O modelo matemático para o reator RCS mostrou se ajustar bem aos dados experimentais.

O estudo de análise de sensibilidade mostrou que para a concentração de NO os parâmetros com maior influência nessa variável são o teor inicial de NO na corrente de alimentação, L e $\alpha$. Em que o efeito do teor de NO é mais acentuado na entrada do leito e os demais parâmetros influenciaram mais na saída do reator.

Ao analisar a concentração de $\mathrm{NH}_{3}$ os parâmetros mais influentes também foram o teor inicial de NO na corrente de alimentação, L e $\alpha$, entretanto apenas L influenciou de forma relevante a concentração de $\mathrm{NH}_{3}$ na saída do reator.

Já para concentração de $\mathrm{SO}_{3}$ o parâmetro mais influente foi a temperatura de alimentação.

\section{Agradecimentos}

Ao Programa de Recursos Humanos da ANP (PRH-28) e ao Laboratório de Controle e Otimização de Processos (LACO-UFPE)

\section{Lista de símbolos}

AV: vazão volumétrica por área superficial geométrica do catalisador

$a$ : espessura média da parede do catalisador monolítico $m$

$C:$ concentração $\frac{m o l}{m^{3}}$

$c_{p}$ : capacidade calorifica $\frac{\mathrm{J}}{\mathrm{kgK}}$

$D_{i}$ : Coeficiente de difusão $\frac{m^{2}}{s}$

$d_{h}$ : diâmetro hidráulico $m$

$h$ : coeficiente de transferência de calor $\frac{W}{m^{2} K}$

$k_{a d s}$ : constante de adsorção da amônia $\mathrm{m}^{3} \mathrm{~mol}^{-1} \mathrm{~s}^{-1}$ 
$\boldsymbol{k}_{\text {des }}:$ constante de dessorção da amônia $\mathbf{1} / \boldsymbol{s}$

$k_{m}$ : coeficiente de transferência de massa gás-sólido em $\frac{m}{s}$

$k$ : condutividade térmica do ar $\frac{W}{m K}$

$L$ : comprimento do canal monolítico em $m$

$R_{j}$ : taxa de reação $\frac{\mathrm{mol}}{\mathrm{m}^{3} \mathrm{~s}}$

$R e$ : número de Reynolds

$r_{a d s}$ : taxa de adsorção $\mathrm{NH}_{3} \frac{\mathrm{mol}}{\mathrm{m}^{3} \mathrm{~s}}$

$r_{a d s}$ : taxa de dessorção $\mathrm{NH}_{3} \frac{\mathrm{mol}}{\mathrm{m}^{3} s}$

$r_{N O}$ : taxa de reação $N O \frac{\mathrm{mol}}{\mathrm{m}^{3} \mathrm{~s}}$

$S_{v}: \frac{1}{d_{h}} m^{-1}$

Sc: número de Shimidt

$T_{g}$ : temperatura da fase gás $\mathrm{K}$

$u$ : velocidade linear do gás em $\frac{m}{s}$

$y_{A}$ : saída do modelo gerada a partir dos dados da matriz de entrada $A$

$y_{B}$ : saída do modelo gerada a partir dos dados da matriz de entrada $B$

$y_{C}{ }^{i}$ : saída do modelo gerada a partir dos dados da matriz de entrada $C^{i}$

$z$ : coordenada axial $m$

$\alpha$ : razão molar $\mathrm{NH}_{3} / \mathrm{NO}$

$\rho$ : densidade $\frac{\mathrm{kg}}{\mathrm{m}^{3}}$

$\varepsilon$ : porosidade do leito

$\Delta H_{j}$ : entalpia de reação $\frac{J}{m o l}$

$\Omega_{N_{3} 3}$ : capacidade catalítica de adsorção da amônia $\frac{m o l}{m^{3}}$

$\Omega_{\mathrm{SO}_{3}}$ : capacidade catalítica de adsorção do $\mathrm{SO}_{3} \frac{\mathrm{mol}}{\mathrm{m}^{3}}$

$\theta_{N H 3}$ : superfície coberta por amônia

$\theta_{\mathrm{SO}_{3}}$ : superfície coberta por $\mathrm{SO}_{3}$

\section{Subscritos}

$g$ : fase gás

$s$ : fase sólida

\section{Referências bibliográficas}

ALENCAR, J. R. B., BOAS, M. C. V. Mecanismos de corrosão esperados nas plantas de SNOX da RNEST. Rio Oil \& Gas Expo and Conference, 2012 Rio de Janeiro, Brasil.

Balanço energético nacional 2014. Disponível em: https://ben.epe.gov.br/downloads/Relatorio Final BEN 2014.pdf

CHEN, C.T; TAN, W.L. 2012. Mathematical modeling, optimal design and control of an SCR reactor for NOx removal. Journal of the Taiwan Institute of Chemical Engineers, 43, 409-419.

DHANUSHKODIA, S.R., MAHINPEY, N., WILSON, M. 2008. Kinetic and 2D reactor modeling for simulation of the catalytic reduction of NOx in the monolith honeycomb reactor. Process safety and environment protection, 86, 303-309. 
FAGHIHI, E. M., SHAMEKHI, A. H. 2010. Development of a neural network model for selective catalytic reduction (SCR) catalytic converter and ammonia dosing optimization using multi objective genetic algorithm. Chemical Engineering Journal, 165, 508-516.

FORZATTI, P., TRONCONI, E., CAVANNA, A., ORSENIGO, C. 1999. Transient Kinetics of SO2 Oxidation Over SCR-DeNOx Monolith Catalysts. Ind. Eng. Chem. Res., 38, 2593-2598.

FORZATTI, P. 2001. Present status and perspectives in de-NOX SCR catalysis. Applied Catalysis A: General. 222, 221-236.

LIETTI, L., NOVA, I., TRONCONI, E., FORZATTI, P. 2000. Unsteady-State kinetics of DeNOx SCR catalysis. Reaction engineering for Pollution Prevention.

NGUYENA, T. D.B., LIMA, Y., EOMB, W.H., KIMB, S.J,. YOO, K.S. 2010. Experiment and CFD simulation of hybrid SNCR-SCR using urea solution in a pilot-scale reactor. Computers and Chemical Engineering, 34, 1580-1589.

Resolução Conama 382. Disponível em: http://www.mma.gov.br/port/conama/legiabre.cfm?codlegi=520

RUGGERI, M. P., NOVA, I., TRONCONI, E. 2012. Experimental and modeling study of the impact of interphase and intraphase diffusional limitations on the DeNOx efficiency of a V-based extruded catalyst for $\mathrm{NH}_{3}-\mathrm{SCR}$ of Diesel exhausts. Chemical Engineering Journal, 207, 57-65.

SALTELLI, A. 2002. Making best use of model evaluations to compute sensitivity índices. Computer Physics Communications, 145, 280-297.

SALTELLI, A., RATTO, M., TARANTOLA, S., CAMPOLONGO, F. 2004. Sensitivity Analysis in Practice A Guide To Assessing Scientific Models, Londres, Wiley.

SALTELli, A., RATTO, M., TARANTOLA, S., CAMPOLONGO, F., GATELLI. D. 2008. Sensitivity Analysis: The Primer, Londres, Wiley.

SALTELLI, A., RATTO, M., TARANTOLA, S., CAMPOLONGO, F. 2012. Sensitivity Analysis for Chemical Models. Chemical Reviews, 112, 1-21.

SANTOS, A., BAHAMONDE, A., SHMID, M., AVILA, P., OCHOA, F. G. 1998. Mass transfer influences on the design of selective catalytic reduction (SCR) monolithic reactors. Chemical Engineering and Processing, 37, 117-124.

SOBOL, I.M. 2001. Global sensitivity indices for nonlinear mathematical models and their Monte Carlo estimates. Mathematics and Computers in Simulation, 55, 271-280.

SOBOL, I.M., TARANTOLA, S., GATELLI. D., KUCHERENKO, S.S., MAUNTZ, W. 2007. Estimating the approximation error when fixing unessential factors in global sensitivity analysis. Reliability Engineering and System Safety, 92, 957-960.

TRONCONI, E., LIETTI, L., MALlOGGI, S., FORZATTI, P. 1996. Experimental and Theoretical Investigation of the Dynamics of the SCR - Denox Reaction. Chemical Engineering Science, 51, 2965-2970.

TRONCONI, E., FORZATTI, P., CAVANNA, A. 1998, Unsteady Analysis of NO Reduction over Selective Catalytic Reduction De-NOx Monolith Catalysts. Ind. Eng. Chem. Res, 37, 2341 2

VOTRUBA, J., MIKUS, O., NGUEN, K., HLAVACEK, V., SKRIVANEK, J. 1975. Heat and mass transfer in honeycomb catalysts II, Chem. Eng. Sci, 30, 201-206. 